\title{
Research Paper: The Effects of Virtual Reality on Upper Limb Function in Chronic Stroke Patients: A Clinical Trial
}

\author{
Hossein Soufi Ahmadi' ${ }^{1}$, Afsoon Hassani Mehraban ${ }^{1,2^{*}}$ (D), Malek Amini ${ }^{1}$ (D), Maryam Sheikhi ${ }^{3}$ (D) \\ 1. Department of Occupational Therapy, School of Rehabilitation Sciences, Iran University of Medical Sciences, Tehran, Iran \\ 2. Rehabilitation Research Center, Iran University of Medical Sciences, Tehran, Iran. \\ 3. Department of Physical Therapy, School of Rehabilitation Sciences, Shiraz University of Medical Sciences, Shiraz, Iran.
}

\begin{tabular}{c|l}
$\begin{array}{c}\text { Use your device to scan } \\
\text { and read the article online }\end{array}$ & $\begin{array}{l}\text { Citation Soufi Ahmadi H, Hassani Mehraban A, Amini M, Sheikhi M. The Effects of Virtual Reality on Upper Limb Function in } \\
\text { Chronic Stroke Patients: A Clinical Trial. Iranian Rehabilitation Journal. 2019; 17(1):81-89. http://dx.doi.org/10.32598/irj.17.1.89 }\end{array}$ \\
dol http://dx.doi.org/10.32598/irj.17.1.89
\end{tabular}

Article info:

Received: 15 Sep 2018

Accepted: 03 Jan 2019

Available Online: 01 Mar 2019

Keywords:

Virtual Reality, Rehabilitation, Stroke, Upper limb function

\section{A B S T RACT}

Objectives: Stroke is one of the main causes of disability and mortality worldwide. Most survivors experience impairments in their upper limb motor function.

Methods: This experimental study was performed as a clinical trial on 30 chronic stroke patients who experienced stroke from 6 to 96 months ago. Patients were non-randomly divided into the intervention (Virtual Reality besides conventional occupational therapy) and control (conventional occupational therapy) groups. Each treatment session lasted for one hour which was divided into conducting conventional occupational therapy techniques and Virtual Reality (VR) for the intervention group, and routine techniques for the control group. The intervention effectiveness was evaluated by the Fugl-Meyer Upper Extremity Scale, Stroke Impact Scale, Chedoke Arm and Hand Activity Inventory, Motricity Index, Modified Ashworth Scale and goniometer. Results were analyzed by SPSS and one-sample Kolmogorov-Smirnov, ShapiroWilk test, Independent Samples t-test and Mann Whitney U test were applied to assess the normality of data and to detect significant differences between study variables.

Results: The results suggested that investigated parameters such as upper limb motor function, muscle tone and the range of motion were significantly different in the intervention group, compared to control group; however, there was no significant changes in none of the group's daily living activities.

Discussion: VR-based computer games in combination with routine occupational therapy interventions could improve upper extremities functional impairments in chronic stroke patients. However, it seems the mechanisms behind the effectiveness of video games and their impact on brain cortex as well as upper limbs function need to be further investigated.

\footnotetext{
* Corresponding Author:

Afsoon Hassani Mehraban, PhD.

Address: Rehabilitation Research Center, Department of Occupational Therapy, School of Rehabilitation Sciences, Iran University of Medical Sciences,

Tehran, Iran.
}

Tel: +98 (21) 22228051

E-mail: mehraban.a@iums.ac.ir.com 


\section{Highlights}

- Computer games can improve upper limb motor function, muscle tone, and the range of motion in stroke patients.

- Using computer games activities did not show any significant effect on activities of daily living.

\section{Plain Language Summary}

Stroke is one of the disabling conditions that can influence the daily living activities and functional performance of survivors. Upper limb dysfunctions are prevalent in most stroke patients. Virtual reality is a new technology that has been recently used for different purposes in the rehabilitation of stroke patients. This technology encourages performing activities in different ways such as playing video games like Nintendo Wii. Virtual reality games can improve upper limb impairments, due to their repetitive activities, voluntary movements performed in various directions and speeds as well as the need to react precisely. It is also, more acceptable for patients, because of the pleasant and encouraging effects. In our study, 15 stroke patients performed virtual reality activities for upper limb rehabilitation. The finding shows the improvement of upper limb motor function, tone, and range of motion in this group. Thus, using this technique with routine occupational therapy can be beneficial for stroke patients.

\section{Introduction}

troke is one of the vascular neurological diseases where vascular damage impairs the brain's blood supply leading to damage and dead of brain cells and tissues $[1,2]$. Common stroke consequences are hemiplegia, sensory impairment, visual, perceptual and cognitive impairments, motor deficits and difficulty in performing targeted movements, and depression [1]. Only $10 \%$ of those surviving from stroke may return to their previous functional level; almost $50 \%$ of them may have desirable functionality with limited help and about $40 \%$ would need help and support for rehabilitation [3].

Studies demonstrated that about $85 \%$ of stroke patients suffer from upper limb impairments. Among this huge number, 40-60\% can use their hands in daily activities and $15 \%$ will regain their functional abilities after a while [4]. Upper limb impairments include impairments in the movement and coordination of arms, hands and fingers and usually limit activities like eating, dressing and washing $[5,6]$. Each of the mentioned disabilities might occur in anyone. Therefore, improving upper limb impairments is a fundamental aim of any rehabilitation program, specially for occupational therapists who specifically work on promoting upper limb function in daily living activities [5-7].

Most of the methods including neurodevelopmental techniques and task-oriented approaches which emphasis on increasing the range of motion, power and endurance are not equipped with measurable and gradable tools [8]. These long-term and repetitive programs could be tedious for patients and cause the lack of adherence, decrease motivation and attention to pursue therapy program, leasing to reduce their efficacy $[4,8]$. All of the mentioned shortcomings have made therapists to replace traditional methods with modern therapeutic computer games.

Virtual Reality (VR) interventions such as video games, which are part of biofeedback techniques, have emerged into the rehabilitation field [9]. VR games can improve upper limb impairments, due to their repetitive activities, voluntary movements performed in various directions, speeds and amplitudes as well as the need to react precisely [10]. Therefore, VR games can successfully lead to motor learning facilitation [11]. Entertaining VR videogames provide feedbacks that encourage patients to participate more in treatment and benefit from their residual functional capacities to achieve success [12].

Nintendo Wii video game has been represented as a secure, accessible and proficient choice for rehabilitation purposes and recovering post-stroke upper extremity impaired functioning. Moreover, VR and videogame applications are groundbreaking impressive technologies that can be efficiently mixed with conventional rehabilitation methods of upper limb impairments after stroke [13, 14]. Upper extremity scores have significantly improved by the use of a Wii device [15].

Nintendo Wii has been suggested as an easily accessible complementary device for improving conventional therapy specially in the subacute stage of stroke rehabili- 
tation in subjects with moderate strength and upper limb functional limitations [15-17]. In addition, VR training could be advantageous in improving the voluntary function of the arm during the acute stage of stroke in subjects participating in an intensive training program [17].

Unlike Nintendo VR system, E-Link device has the capacity for measuring as well as analyzing upper extremity function. Additionally, it has various handles that enable the practitioner to train and rehabilitate the patients for operating more diverse movements [18]. Despite the extensive use of the computer in human life, its application is very limited in rehabilitation. The majority of studies in this field have not described a precise protocol or have used commercial devices which are not designed specifically for therapeutic purposes. To our knowledge, no study has investigated the efficacy of using VR (E-Link) in stroke patients in Iran. Thus, we investigated the effectiveness of VR on upper arm functioning in chronic stroke patients.

We assumed that the unique characteristics of this technology could be used as an impressive tool for improving upper limb functioning in the subjects with motor deficits. The therapeutically designed system experimented in this study was adopted for the first time to provide intervention and evaluation, concurrently. In this study, we compared 12 sessions of Traditional Occupational Therapy (TOT), with the same number of sessions combining TOT with VR E-Link on the upper extremity functioning in stroke patients to realize which could be more beneficial.

\section{Methods}

\section{Study design}

The present study was a non-randomized controlled trial. The samples were selected among patients referring to the occupational therapy laboratory of Iran University of Medical Sciences. Patients were divided nonrandomly and according to their sequence of referring to the lab. There were 15 subjects (10 right hemiplegia and 5 left hemiplegia) in the intervention group (traditional occupational therapy methods accompanied by VR) and 15 (10 right hemiplegia and 5 left hemiplegia) in the control group (only traditional occupational therapy methods). This clinical trial was registered (IR.IUMS.REC1395.9411355008) and approved by the Ethics Committee of the Rehabilitation Center of Iran University of Medical Sciences. Written informed consent was obtained from all participants before their enrollment to the study.

\section{Study participants}

This study was implemented in the School of Rehabilitation Sciences (Iran University of Medical Sciences) in 2017. All study participants suffered from stroke for a minimum time of 6 months to a maximum time of 96 months. The inclusion criteria were suffering from stroke for at least 6 months due to the diagnosis of a neurologist, Brunnstorm's upper limb function of $\geq 4$, a cognitive function score of 21 by Mini Mental Status Examination (MMSE), the lack of any other neurological disorders according to their medical records, their family or their physicians report, achieving maximum score of $1+$ in Modified Ashworth Scale and having normal vision (not having any visual impairments such as hemianopsia).

\section{Study procedure}

\section{Assessment tools}

The Fugl-Meyer Upper Extremity (FMUE) Scale is a performance-based scale broadly used to measure impairments in stroke patients. Reflex activity, movement control and muscle strength of upper extremity are the main parameters assessed in post-stroke hemiplegic patients by this tool. The FMUE includes 33 items. Each item is scored 0 if the participant fails to perform it, 1 if the subject partially performs the function and 2 for fulfilling the task, ideally. The whole test takes almost 30 minutes to be completed and has revealed acceptable reliability, validity and responsiveness in stroke evaluations. A distinguished feature of this test is no needs for specialized equipment or preparation and could be administered in a variety of occasions as well as long lasting assessments [19].

The Chedoke Arm and Hand Activity Inventory (CAHAI) has been used for assessing the recovery of poststroke upper limb function since 2004 when Barreca et al. first developed it to investigate the recovery of paretic limb in this category of patients. Fundamental objectives of the test are as follows: to discriminate between various dysfunctions of upper limb, predict functional recovery in the affected upper limb, quantify upper limb functional changes, determine the critical results of these changes in stroke survivors and be a valid tool for guiding treatment. The test comprises of 13 real life functional tasks. Scoring of all 13 tasks is based on a 7-point quantitative scale. The total scale would be at a minimum amount of 13 to a maximum amount of 91, which higher scores reveal greater abilities. The total amount of time required for test administration is $25 \mathrm{~min}$ [20]. 
The Stroke Impact Scale (SIS) is a stroke-specific self-report scale with 2 versions. The second version has 64 items that are categorized into 8 domains (strength, hand function, Activities of Daily Living (ADL), communication, emotion, mobility, memory and thinking, and participation). Maximum achievable point for each item is 5 . The scoring is based on the level of difficulty experienced by patients while performing each task in the previous week. The higher total score is representative of performing the task without any difficulties [21]. We used items 7 (SIS 1) and 9 (SIS 2) of this questionnaire which assess daily living activities, to evaluate our results.

The Modified Ashworth scale was originally invented to investigate the impact of an anti-spastic drug on Multiple Sclerosis patients. The scale is rated by the examiner to assess the amount of perceived resistance or muscle tone while moving the limb through the full range of motion. The original version consisted of 5 grades from 0 to 4 ; however, in 1987, the scale was reworded to enhance the sensitivity of it and one grade (1+) was added [22].

The Mini-Mental State Examination (MMSE) was first designed as a screening tool to quantitatively investigate cognitive impairments and record its changes over time. The MMSE has 11 simple questions or tasks, classified in 7 cognitive categories. These 7 domains are orientation to place and time, registration of 3 words, attention and calculation, recall of 3 words, as well as language and visual construction. The test completion takes about 10 minutes provided that it is conducted by an expert interviewer. The test explores patients' cognitive performance and has a total score of 30 . A score of 23 or 24 is generally accepted as the cutoff point which indicates cognitive impairment presence. Impairment levels are classified as none (24 to 30 ), mild (18 to 24$)$, and severe (0 to 17) [23].

The Motricity Index is applied for grading muscle strength based on patients' ability to activate a muscle group, to move a limb segment within the range of motion, as well as resist against examiner's force. The validity of this index for upper limb function is confirmed by the close interrelation between its components and its correlation with grip strength and upper extremity function [24].

The Goniometer is a useful instrument to measure joints' angle and the range of motion for either active or passive movements [25].

\section{Intervention}

A 4-week continuous program was designed for all of the study participants. Both of the control and intervention groups received 1-hour sessions of three times per week with the exception that the control group only received TOT for the 1-hour sessions; however, the time was divided for the intervention group to 40 minutes of VR E-Link and 20 minutes of TOT. All the interventions and data collections were performed by one trained examiner to increase the accuracy. Our intervention was mainly focused on the function of the distal part of the upper extremity.

\section{VR E-link}

The VR system used in the current study was E-Link (Evaluation and Exercise, Biometrics Ltd systems, version 6). The advantage of this computer system is the capacity to evaluate several times and applies treatment exercises within defined games with the purpose of specific usage in rehabilitation. The loading and resistance rates are also adjustable in accordance with the patients' disabilities. Additionally, this system has the capacity to evaluate the active and passive ranges of motion for every single functional movement.

The E-link categorizes training games for desired movements as follow: forearm supination/pronation and wrist flexion/extension in the vertical and horizontal planes. All the practices in this study were simple, colorful and exciting, such as driving, playing basketball, space shooting and so on. The difficulty and complexity levels of games were different and adjusted based on the individuals' ability. At the beginning of each session, movement functions were explained and patients learnt how to play and work with the system, depending on the functionality of hand, joystick and games.

Catch balls, Find space, Load ship and Solar eclipse were played by cylindrical joysticks and games like Maze, Wipe out, Driving and Space shooting were played by spherical joysticks for improving wrist extension and forearm supination, respectively. The videogames were run at various times according to patients' interest and movement restrictions. If needed, participants were given a break during playing. The mentioned movements were selected to achieve specific task-based goals in real life situations by visual feedback. The comfortability of all parts like joysticks have been medically tested and approved. 
TOT

The TOT intervention included strengthening, the range of motion, table top activities and training activities of daily living for the more involved limb. TOT interventions included the similar movements of the distal part of upper extremity in VR E-Link group, to maximize the integrity between the two groups. The difficulty of the intervention was changed considering the level of participant's performance in each group, as well.

Finally, the functionality of the upper limb, muscle strength, movement range, and the quality of the daily activities were reassessed after the completion of intervention.

\section{Statistical analysis}

Statistical analysis was performed by SPSS after the obtained data were encoded. Data normality was examined by the Shapiro-Wilk test, and Mann-Whitney U test was used to compare the efficacy of interventions.

\section{Results}

The mean age of the control and intervention groups were 55.26 and 55.23, with the maximum age of 80 and 72 and a minimum of 39 and 36 years, respectively. The maximum and minimum time passed after the stroke was 50 and 11 months for the control group and 96 and 7 months for the intervention group. The MMSE average scores were 27.09 and 28.20 in the control and interven- tion groups, respectively. A maximum score of mental status testing for both groups was 30 ; the minimum score for the control group was 22 and for the intervention group was 23. Qualitative demographic variables are presented in Table 1.

\section{Analytic results}

Data analysis of the functionality variables of upper limb, movement range and muscle tone revealed significant differences in the intervention group, compared to the controls $(\mathrm{P}<0.05)$. The results of data analysis for both TOT and VR E-Link group are listed in Table 2 and 3. Pre-test and post-test comparisons in both groups were statistically significant, indicating that both treatment protocols were effective and improved the upper limb function. The obtained results demonstrated that VR E-Link in combination with TOT was more efficient in Fugl-Meyer $(\mathrm{P}<0.001)$, Motoricity Index $(\mathrm{P}<0.001)$ and Chedoke Test $(\mathrm{P}<0.001)$, compared to the control group.

Regarding the intervention group, Fugl-Meyer wrist part $(\mathrm{P}<0.001)$ and Fugl-Meyer hand part $(\mathrm{P}=0.001)$ were statistically significant, compared to the control group. The forearm supination/pronation and wrist flexion/extension movement ranges measured by goniometer were significantly different with the $\mathrm{P}<0.001$ for both variables. The measures of daily activity revealed no significant difference in any group $(\mathrm{P}>0.05)$. The data on daily living activities reviewed by the Stroke Impact Scale suggested no significant difference between the

Table 1. Qualitative demographic variables

\begin{tabular}{|c|c|c|c|c|c|}
\hline \multirow{3}{*}{ Variables } & & \multicolumn{4}{|c|}{ Group } \\
\hline & & \multicolumn{2}{|c|}{ Intervention } & \multicolumn{2}{|c|}{ Control } \\
\hline & & No. & Frequency (\%) & No. & Frequency (\%) \\
\hline \multirow{2}{*}{ Gender } & Male & 11 & 73.33 & 9 & 60 \\
\hline & Female & 4 & 26.66 & 6 & 40 \\
\hline \multirow{2}{*}{ Affected side } & Right & 10 & 66.66 & 10 & 66.66 \\
\hline & Left & 5 & 33.33 & 5 & 33.33 \\
\hline \multirow{2}{*}{ Marriage status } & Married & 14 & 93.33 & 15 & 100 \\
\hline & Single & 1 & 6.66 & 0 & 0 \\
\hline \multirow{2}{*}{ Dominant side } & Right & 13 & 86.66 & 13 & 86.66 \\
\hline & Left & 2 & 13.33 & 2 & 13.33 \\
\hline
\end{tabular}


Table 2. Analytical statistics of variables with non-normal distribution

\begin{tabular}{|c|c|c|c|c|c|c|}
\hline Variables & Group & No. & Mean Differences & Difference Changes & Z & $\mathbf{P}$ \\
\hline \multirow{3}{*}{ MAS forearm supination } & Intervention & 15 & 0.33 & \multirow{3}{*}{0.16} & \multirow{3}{*}{1.7} & \multirow{3}{*}{0.001} \\
\hline & & & & & & \\
\hline & Control & 15 & 0.16 & & & \\
\hline \multirow{3}{*}{ MAS wrist extension } & Intervention & 15 & 0.33 & \multirow{3}{*}{0.16} & \multirow{3}{*}{1.7} & \multirow{3}{*}{0.001} \\
\hline & & & & & & \\
\hline & Control & 15 & 0.16 & & & \\
\hline \multirow{3}{*}{ SIS1 } & Intervention & 15 & 0.00 & \multirow{3}{*}{1.66} & \multirow{3}{*}{2.40} & \multirow{3}{*}{0.01} \\
\hline & & & & & & \\
\hline & Control & 15 & 1.66 & & & \\
\hline \multirow{3}{*}{ SIS2 } & Intervention & 15 & 0.80 & \multirow{3}{*}{2.26} & \multirow{3}{*}{2.11} & \multirow{3}{*}{0.03} \\
\hline & & & & & & \\
\hline & Control & 15 & 1.46 & & & \\
\hline \multirow{3}{*}{ FMA (wrist) } & Intervention & 15 & 1.33 & \multirow{3}{*}{1.93} & \multirow{3}{*}{3.66} & \multirow{3}{*}{$<0.001$} \\
\hline & & & & & & \\
\hline & Control & 15 & 1.40 & & & \\
\hline \multirow{3}{*}{ FMA (hand) } & Intervention & 15 & 3.20 & \multirow{3}{*}{0.73} & \multirow{3}{*}{1.61} & \multirow{3}{*}{0.001} \\
\hline & & & & & & \\
\hline & Control & 15 & 2.46 & & & \\
\hline \multirow{3}{*}{ FMA (total score) } & Intervention & 15 & 6.53 & \multirow{3}{*}{2.66} & \multirow{3}{*}{3.16} & \multirow{3}{*}{0.001} \\
\hline & & & & & & \\
\hline & Control & 15 & 3.86 & & & \\
\hline \multirow{3}{*}{ ROM forearm supination } & Intervention & 15 & 7.66 & \multirow{3}{*}{6.66} & \multirow{3}{*}{4.50} & \multirow{3}{*}{$<0.001$} \\
\hline & & & & & & \\
\hline & Control & 15 & 1.00 & & & \\
\hline & Intervention & 15 & 7.66 & & & \\
\hline ROM wrist extension & & & & 5.33 & 3.93 & $<0.001$ \\
\hline & Control & 15 & 2.33 & & & \\
\hline
\end{tabular}

Iranian Rehabilitation Journal

study groups $(\mathrm{P}>0.05)$. Chedoke Test and Motricity Index had normal data distribution, while others had not (Tables 2 and 3 ).

\section{Discussion}

The present study examined the effect of VR technology (by the use of E-Link) on the upper limb function in stroke patients. Our results revealed that VR technology could improve upper extremity function in chronic stroke patients. Patients reported no pain or discomfort after the intervention. Despite their lack of experience in working with VR, they could learn to make neces-

Table 3. Analytical statistics of variables with normal distribution

\begin{tabular}{ccccccc}
\hline Variables & Group & No. & Mean Differences & Difference Changes & t & P \\
\hline \multirow{2}{*}{ Chedoke } & Intervention & 15 & 6.73 & 4.2000 & 4.31 & $<0.001$ \\
& Control & 15 & 2.53 & & & \\
Motricity & Intervention & 15 & 8.83 & 6.2000 & 3.56 & 0.001 \\
& Control & 15 & 2.66 & & & \\
\hline
\end{tabular}


sary changes in a short time. Fugl-Meyer (wrist and hand parts), Motricity Index (arm score part) and Chedoke Arm and Hand Activity Inventory were used to assess upper limb function status.

According to the increase in Fugl-Meyer, CAHAI and Motricity Index scores, the upper limb function skill was generally improved. Goniometer measures on a range of motion changes, compared to the baseline, revealed the efficacy of intervention in the VR E-Link group. This may be explained by the similarity of selected exercises of VR with the necessities of volitional forward movement. In addition, significant differences of Modified Ashworth Scale scores indicated the efficacy of our intervention on forearm supination/pronation and wrist flexion/extension muscle tone. Although there were no specific exercises for muscle tone reduction, the increased scores could be the results of improvements in the selfengagement of patients in motor control and their abilities in performing voluntary movements. These results are consistent with the previous studies; however, most of the previous studies used Wii system.

Carregosa et al. reported that after Nintendo Wii intervention, motor learning retention was observed in patients, which indicates obtaining a sustained benefit through the technique. Therefore, sub-item and total score values related to the upper limb motor function by the Fugl-Meyer scale and functional capacity by the SF36 questionnaire were sustained. This finding indicated that the therapeutic consequences would possibly remain by the pass of time [26].

Brunner et al. indicated similar results in sub-acute phase of stroke patients [27]. Kong et al. presented the efficacy of Nintendo Wii in facilitating upper limb recovery in stroke patients, in companion with conventional methods. They compared the results of 12 sessions of upper limb exercises by Nintendo Wii gaming, and conventional therapy within 3 weeks. They indicated that none of the methods were effective in promoting upper limb motor recovery, in comparison to control group [28].

Park et al. has stated that game-based VR movement therapy may be helpful for upper extremity functional recovery. They used the Fugl-Meyer assessment tool and Box and Block Test as well as the quality of movement subscale of the motor activity log [29]. Studies examined stroke patients in the acute phase. However, due to the spontaneous capability for recovery of patients in this phase, their results are not reliable. Moreover, assessment tools used in previous studies were not subtle enough to be used in occupational therapy assessments. Stroke Impact Scale was used to examine patients ADL and IADL status. This scale revealed more significant changes in the control group.

Kwon et al. the indicated no benefit for intensive VR programs combined with conventional techniques in the acute phase of stroke, compared to conventional techniques alone. No significant difference was observed in ADL performance and upper extremity function [16]. In our study, the comparison of pre-treatment and posttreatment values for both groups were significant, except for SIS. Our results highlight the effectiveness of implementing VR practices simultaneously with traditional occupational therapy techniques to improve upper limb function in stroke survivors.

The results of the current study could be explained by the compact and targeted VR practices, occupational therapy interventions, neuromuscular system flexibility and consequent movement improvements in both groups of participants. Percentage comparison of changes in tests suggested that VR along with occupational therapy improved Fugl-Meyer, Motricity Index and Chedoke test scores of upper limbs, except for SIS test in the intervention group. The improvement of the measures in the intervention group is because of the advantageous effects of traditional occupational therapy practices along with VR practices. Daily living activities did not significantly affect the groups $[5,30,31]$. Additionally, the high satisfaction rate of participants indicates the joyful and motivational nature of such activities accompanied by traditional treatments.

\section{Conclusion}

This study suggested that VR and common occupational therapy techniques could improve upper limb function in post-stroke patients who are in their chronic phase. Therefore, we suggest that this technology be successfully accompanied by traditional techniques to gain more efficient results in upper extremity functional activity among stroke patients.

\section{Ethical Considerations}

\section{Compliance with ethical guidelines}

This clinical trial was registered (IR.IUMS. REC1395.9411355008) and approved by the Ethics Committee of the Rehabilitation Center of Iran University of Medical Sciences. Written informed consent was 
obtained from all participants before their enrollment to the study.

Funding

The present paper was extracted from the MSc. thesis of the first author, in Department of Occupational Therapy, School of Rehabilitation Sciences, Iran University of Medical Sciences.

\section{Authors contributions}

Conception, design and writing: Afsoon Hassani Mehraban; planning: Malek Amini; Implementation of intervention: Hossein Soufi Ahmadi; and helping in writing: Maryam sheikhi.

\section{Conflict of interest}

The authors declared no conflict of interest.

\section{References}

[1] Radomski MV, Latham CAT. Occupational therapy for physical dysfunction. Philadelphia: Lippincott Williams \& Wilkins; 2008. [PMCID]

[2] Yen CY, Lin KH, Hu MH, Wu RM, Lu TW, Lin CH. Effects of Virtual Reality- augmented balance training on sensory organization and attentional demand for postural control in people with parkinson disease: A randomized controlled trial. Physical Therapy. 2011; 91(6):862-74. [DOI:10.2522/ ptj.20100050] [PMID]

[3] Kernan WN, Viscoli CM, Brass LM, Broderick JP, Brott T, Feldmann E, et al. Phenylpropanolamine and the risk of hemorrhagic stroke. New England Journal of Medicine. 2000; 343(25):182632. [DOI:10.1056/NEJM200012213432501] [PMID]

[4] Krug G, McCormack G. Occupational therapy: Evidencebased interventions for stroke. Missouri Medicine. 2009; 106(2):145-9. [PMID]

[5] Lee JH, Ku J, Cho W, Hahn WY, Kim IY, Lee SM, et al. A Virtual Reality system for the assessment and rehabilitation of the activities of daily living. CyberPsychology \& Behavior. 2003; 6(4):383-8. [DOI:10.1089/109493103322278763] [PMID]

[6] Hitzeman N, Reiss M. Occupational therapy improves activities of daily living after stroke. American Family Physician. 2007; 75(11):1651-2. [PMID]

[7] Jarvis K, Reid G, Edelstyn N, Hunter S. Development of the occupational therapy stroke arm and hand record: An upper limb treatment schedule. British Journal of Occupational Therapy. 2014; 77(3):126-33. [DOI:10.4276/03080221 4X13941036266469]
[8] Dobkin BH. Strategies for stroke rehabilitation. The Lancet Neurology. 2004; 3(9):528-36. [DOI:10.1016/S14744422(04)00851-8]

[9] Standen P, Threapleton K, Richardson A, Connell L, Brown D, Battersby S, et al. A low cost Virtual Reality system for home based rehabilitation of the arm following stroke: A randomised controlled feasibility trial. Clinical Rehabilitation. 2017; 31(3):340-50. [DOI:10.1177/0269215516640320] [PMID] [PMCID]

[10] Adams RJ, Lichter MD, Ellington A, White M, Armstead K, Patrie JT, et al. Virtual activities of daily living for recovery of upper extremity motor function. IEEE Transactions on Neural Systems and Rehabilitation Engineering. 2018; 26(1):25260. [DOI:10.1109/TNSRE.2017.2771272] [PMID]

[11] Held JP, Luft AR, Veerbeek JM. Encouragement-induced real-world upper limb use after stroke by a tracking and Feedback device: A study protocol for a multi-center, assessorblinded, randomized controlled trial. Frontiers in Neurology. 2018; 9:13. [DOI:10.3389/fneur.2018.00013] [PMID] [PMCID]

[12] Dascal J, Reid M, Ishak WW, Spiegel B, Recacho J, Rosen B, et al. Virtual Reality and medical inpatients: A systematic review of randomized, controlled trials. Innovations in Clinical Neuroscience. 2017; 14(1-2):14-21. [PMID] [PMCID]

[13] Saposnik G, Mamdani M, Bayley M, Thorpe KE, Hall J, Cohen LG, et al. Effectiveness of Virtual Reality Exercises in STroke rehabilitation (EVREST): Rationale, design, and protocol of a pilot randomized clinical trial assessing the Wii gaming system. International Journal of Stroke. 2010; 5(1):47-51. [DOI:10.1111/j.1747-4949.2009.00404.x] [PMID] [PMCID]

[14] Saposnik G, Levin M, Stroke Outcome Research Canada (SORCan) Working Group. Virtual Reality in stroke rehabilitation: A meta-analysis and implications for clinicians. Stroke. 2011; 42(5):1380-6. [DOI:10.1161/STROKEAHA.110.605451] [PMID]

[15] Joo LY, Yin TS, Xu D, Thia E, Chia PF, Kuah CWK, et al. A feasibility study using interactive commercial off-the-shelf computer gaming in upper limb rehabilitation in patients after stroke. Journal of Rehabilitation Medicine. 2010; 42(5):43741. [DOI:10.2340/16501977-0528] [PMID]

[16] Kwon JS, Park MJ, Yoon IJ, Park SH. Effects of Virtual Reality on upper extremity function and activities of daily living performance in acute stroke: A double-blind randomized clinical trial. Neuro Rehabilitation. 2012; 31(4):379-85. [PMID]

[17] Mouawad MR, DoustCG,MaxMD, McNulty PA. Wii-based movement therapy to promote improved upper extremity function post-stroke: A pilot study. Journal of Rehabilitation Medicine. 2011; 43(6):527-33. [DOI:10.2340/16501977-0816] [PMID]

[18] Monfared NRS, Mehraban AH, Boroumand S. Effectiveness of videogames on balance and fear of falling in chronic stroke patient. Iranian Rehabilitation Journal. 2015; 13(1):68-74.

[19] Singer B, Garcia-Vega J. The fugl-meyer upper extremity scale. Journal of Physiotherapy. 2017; 63(1):53. [DOI:10.1016/j. jphys.2016.08.010] [PMID]

[20] Barreca S, Gowland C, Stratford P, Huijbregts M, Griffiths J, Torresin W, et al. Development of the chedoke arm and hand activity inventory: theoretical constructs, item generation, and selection. Topics in Stroke Rehabilitation. 2004 11(4):31-42. [DOI:10.1310/JU8P-UVK6-68VW-CF3W] [PMID] 
[21] Duncan PW, Wallace D, Lai SM, Johnson D, Embretson S, Laster LJ. The stroke impact scale version 2.0: Evaluation of reliability, validity, and sensitivity to change. Stroke. 1999; 30(10):2131-40. [DOI:10.1161/01.STR.30.10.2131] [PMID]

[22] Damiano DL, Quinlivan JM, Owen BF, Payne P, Nelson $\mathrm{KC}$, Abel MF. What does the ashworth scale really measure and are instrumented measures more valid and precise? Developmental Medicine and Child Neurology. 2002; 44(2):1128. [DOI:10.1017/S0012162201001761] [PMID]

[23] Tombaugh TN, McIntyre NJ. The mini-mental state examination: A comprehensive review. Journal of the American Geriatrics Society. 1992; 40(9):922-35. [DOI:10.1111/j.1532-5415.1992.tb01992.x]

[24] Bohannon RW. Motricity index scores are valid indicators of paretic upper extremity strength following stroke. Journal of Physical Therapy Science. 2001; 11(2):59-61. [DOI:10.1589/ jpts.11.59]

[25] Gajdosik RL, Bohannon RW. Clinical measurement of range of motion: Review of goniometry emphasizing reliability and validity. Physical Therapy. 1987; 67(12):1867-72. [DOI:10.1093/ptj/67.12.1867]

[26] Carregosa AA, dos Santos LRA, Masruha MR, Coêlho MLdS, Machado TC, Souza DCB, et al. Virtual rehabilitation through nintendo Wii in poststroke patients: Follow-up. Journal of Stroke and Cerebrovascular Diseases. 2018; 27(2):494-8 [DOI:10.1016/j.jstrokecerebrovasdis.2017.09.029] [PMID]

[27] Brunner I, Skouen JS, Hofstad H, Aßmus J, Becker F, Sanders AM, et al. Virtual Reality Training for Upper Extremity in Subacute Stroke (VIRTUES): A multicenter RCT. Neurology. 2017; 89(24):2413-21. [DOI:10.1212/WNL.0000000000004744] [PMID]

[28] Kong KH, Loh YJ, Thia E, Chai A, Ng CY, Soh YM, et al. Efficacy of a Virtual Reality commercial gaming device in upper limb recovery after stroke: A randomized, controlled study. Topics in Stroke Rehabilitation. 2016; 23(5):333-40. [DOI:10.10 80/10749357.2016.1139796] [PMID]

[29] Park JH, Park JH. The effects of game-based Virtual Reality movement therapy plus mental practice on upper extremity function in chronic stroke patients with hemiparesis: A randomized controlled trial. Journal of Physical Therapy Science. 2016; 28(3):811-5. [DOI:10.1589/jpts.28.811] [PMID] [PMCID]

[30] Lee G. Effects of training using video games on the muscle strength, muscle tone, and activities of daily living of chronic stroke patients. Journal of Physical Therapy Science. 2013; 25(5):595-7. [DOI:10.1589/jpts.25.595] [PMID] [PMCID]

[31] Perez-Marcos D, Chevalley O, Schmidlin T, Garipelli G, Serino A, Vuadens P, et al. Increasing upper limb training intensity in chronic stroke using embodied Virtual Reality: A pilot study. Journal of Neuroengineering and Rehabilitation. 2017; 14(1):119. [DOI:10.1186/s12984-017-0328-9] [PMID] [PMCID] 